\title{
Polarization-Dependent Loss Impact on Coherent Optical Systems in Presence of Fiber Nonlinearity
}

\author{
Nicola Rossi, Paolo Serena, Member, IEEE, and Alberto Bononi, Senior Member, IEEE
}

\begin{abstract}
We investigate the interaction of polarizationdependent loss (PDL) with Kerr nonlinearities by decoupling single- and cross-channel nonlinear distortions in a homogeneous 100-G polarization division multiplexing quadrature phase shift keying (PDM-QPSK) and a hybrid 100-G PDM-QPSK/10-G on/off keying system. Because of the decoupling method, we provide reasons for the interaction, using basic comprehensive examples. Both average Q-factor and its distribution are investigated in both dispersion-managed and dispersion-unmanaged links, showing that realistic PDL values affect outage probabilities notwithstanding a negligible average penalty.
\end{abstract}

Index Terms-Polarization-dependent loss (PDL), fiber nonlinear optics, outage probability.

\section{INTRODUCTION}

D IGITAL signal processing (DSP) has been the breakthrough for optical communication systems that enabled solutions for $100 \mathrm{~Gb} / \mathrm{s}$ long-haul transmissions. DSP units can compensate for linear impairments like chromatic dispersion (CD) and polarization mode dispersion (PMD), while only partial mitigation can be performed on nonlinear distortion and polarization dependent loss (PDL). Unlike PMD, PDL is a non-unitary linear transformation, hence does impact also the amplified spontaneous emission (ASE) noise cumulated along the link, which experiences a different PDL effect than the transmitted signal [1]. As a result, PDL shows up as a polarization-dependent signal to noise ratio (SNR) at the receiver. Early studies analyzed the impact of PDL in polarization division multiplexed quadrature phase shift keying (PDM-QPSK) systems operating in the linear regime, e.g. [2], [3], and only recently the interplay between PDL and the nonlinear Kerr effect has come to the stage [4]-[7].

In this letter, for the first time to our knowledge, we numerically investigate the interaction between PDL and Kerr effect by decoupling the fiber nonlinearities, namely, self phase modulation (SPM), scalar cross phase modulation (XPM) and cross polarization modulation (XPolM) [8]. By means of simple setups we explain the reasons of such interaction. Moreover, we quantify the impact of PDL on both the average performance and its statistical distribution by considering three possible scenarios. In the first case we transmitted over a

Manuscript received October 4, 2013; revised November 8, 2013; accepted November 27, 2013. Date of publication December 3, 2013; date of current version January 21, 2014.

The authors are with the Department of Information Engineering, Università degli Studi di Parma, Parma 43124, Italy (e-mail: rossi_n@tlc.unipr.it; paolo.serena@unipr.it; alberto.bononi@unipr.it).

Color versions of one or more of the figures in this letter are available online at http://ieeexplore.ieee.org.

Digital Object Identifier 10.1109/LPT.2013.2293595

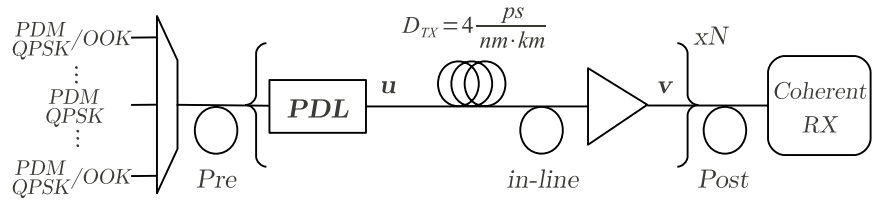

Fig. 1. The simulated system with lumped PDL along the link.

dispersion managed (DM) link a homogeneous wavelength division multiplexed (WDM) system with non-return-to-zero (NRZ) $100 \mathrm{~Gb} / \mathrm{s}$ PDM-QPSK channels. In the second case we repeated the same test when surrounding the reference $100 \mathrm{~Gb} / \mathrm{s}$ PDM-QPSK channel with $10 \mathrm{~Gb} / \mathrm{s}$ NRZ on off keying (OOK) neighbors, giving a hybrid system. In the third case we transmitted over a dispersion-unmanaged (DU) link a WDM system with $100 \mathrm{~Gb} / \mathrm{s}$ PDM-QPSK channels.

\section{NumericAl SETUP}

We used the the open source software Optilux [9] to simulate the system depicted in Fig. 1, where PDL was implemented by a lumped element placed before the transmission fiber. Its input/output field relation is expressed as:

$$
\begin{aligned}
{\left[\begin{array}{l}
u_{X}^{(i)} \\
u_{Y}^{(i)}
\end{array}\right]=} & {\left[\begin{array}{cc}
\sqrt{1+\Gamma} & 0 \\
0 & \sqrt{1-\Gamma}
\end{array}\right]\left[\begin{array}{cc}
\cos \vartheta_{i} & \sin \vartheta_{i} \\
-\sin \vartheta_{i} & \cos \vartheta_{i}
\end{array}\right] } \\
& \times\left[\begin{array}{cc}
\cos \varepsilon_{i} & j \sin \varepsilon_{i} \\
j \sin \varepsilon_{i} & \cos \varepsilon_{i}
\end{array}\right]\left[\begin{array}{c}
v_{X}^{(i-1)} \\
v_{Y}^{(i-1)}
\end{array}\right]
\end{aligned}
$$

where $\mathbf{v}^{(i)}=\left[v_{X}^{(i)}, v_{Y}^{(i)}\right]^{T}$ is the noisy field at the end of the $i$ th span, $i=1, \ldots, N$, where subscripts $\mathrm{X}$ and $\mathrm{Y}$ indicate the two polarizations. $\Gamma$ is the normalized PDL coefficient while $\left(\vartheta_{i}, \varepsilon_{i}\right)$ are azimuth and ellipticity of the $i$ th PDL element eigenvector. $j$ is the imaginary unit.

In the DM case the optical link was composed of $N=20$ spans of non-zero dispersion shifted fiber (NZDSF) (length $100 \mathrm{~km}$, attenuation $0.2 \mathrm{~dB} / \mathrm{km}$, dispersion $4 \mathrm{ps} / \mathrm{nm} / \mathrm{km}$, nonlinear index $\gamma=1.51 / \mathrm{W} / \mathrm{km}$, no PMD) with residual dispersion per span of $30 \mathrm{ps} / \mathrm{nm}$ and Pre-compensation of $-345 \mathrm{ps} / \mathrm{nm}$. The DU link was identical, except for a total of $N=25$ spans with neither in-line compensation nor Pre-compensation. In all cases, after the link, a postcompensating fiber set the overall cumulated dispersion to zero. The propagation within the optical fibers was numerically solved by the split-step Fourier algorithm as a concatenation of linear and nonlinear steps. Each nonlinear step was implemented by activating the nonlinearity of interest, namely SPM, $\mathrm{XPM}$ or XPolM, as in [8]. 

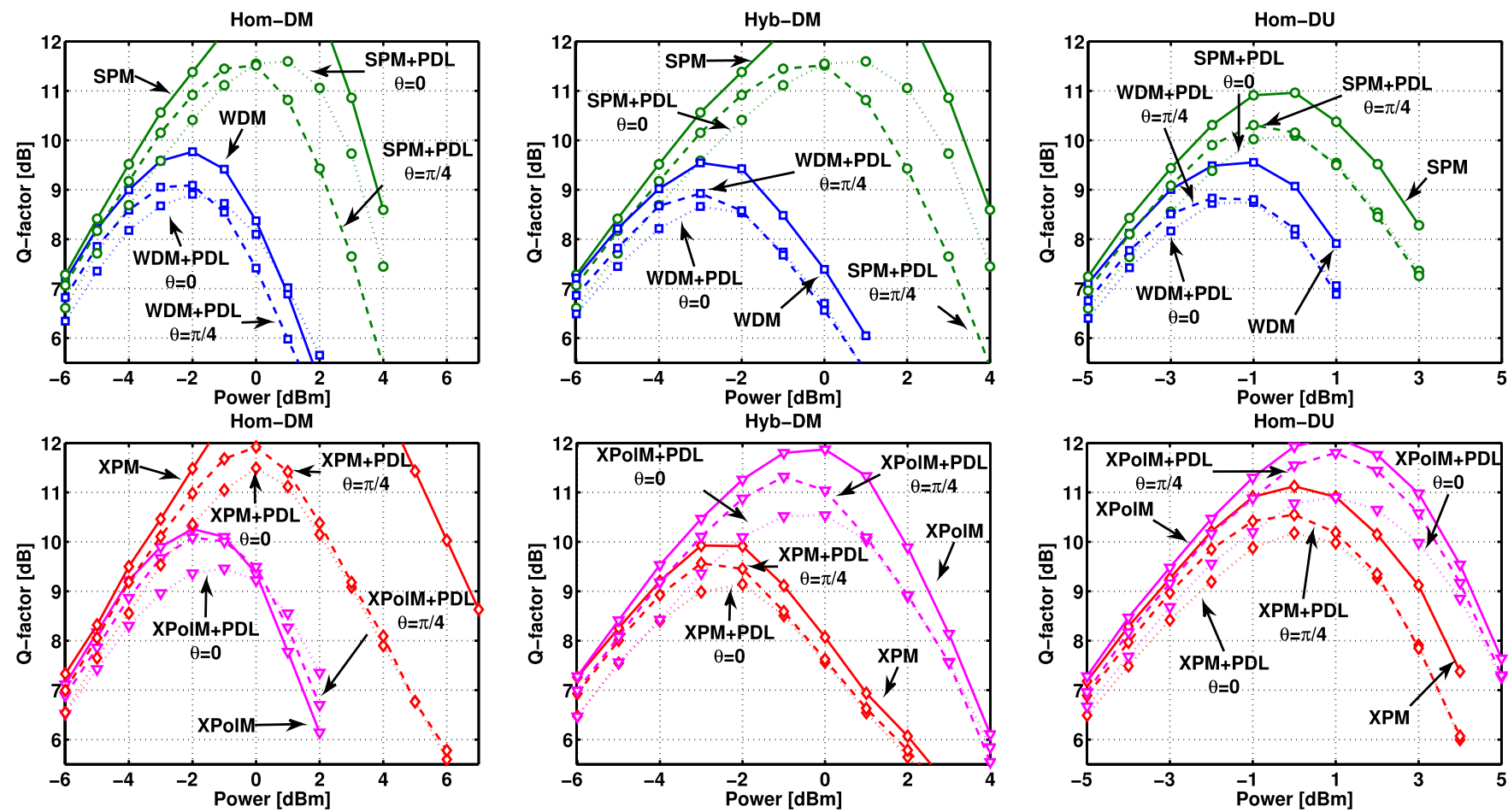

Fig. 2. Average performance for the homogeneous setup on the $20 \times 100 \mathrm{~km}$ DM link (left column), for the hybrid setup on the $20 \times 100 \mathrm{~km}$ DM link (center column) and for the homogeneous setup on the $25 \times 100 \mathrm{~km}$ DU link (right column), without PDL (solid lines) or with a total PDL of $\rho=5 \mathrm{~dB}$, $\varepsilon=0$. (Top row) Propagation of single-channel or WDM. (Bottom row) Propagation with only selected cross-channel impairments.

The nonlinear signal-noise interaction along the link was accounted for by flat-gain noisy amplifiers with $7 \mathrm{~dB}$ noise figure. The WDM comb consisted of 19 channels, with $50 \mathrm{GHz}$ spacing. The central channel was a $112 \mathrm{~Gb} / \mathrm{s}$ PDM-QPSK, surrounded by either $112 \mathrm{~Gb} / \mathrm{s}$ PDM-QPSK channels or by $10 \mathrm{~Gb} / \mathrm{s}$ OOK channels. In the homogeneous DM case we used for each PDM-QPSK channel 1024 random symbols, in the hybrid DM case 840, while in the homogeneous DU case 2048. The DSP-based receiver performed polarization recovery by a 2-samples-per-symbol data-aided least-squares equalizer with 7 taps and carrier phase estimation through the Viterbi\&Viterbi algorithm with 15 taps. The performance was measured in terms of the Q-factor obtained by inverting the bit error rate (BER) of the central channel, estimated from Monte Carlo (MC) simulation stopped after counting at least 300 errors.

\section{Average Q-FACTOR}

Fig. 2 (left column) shows the average Q-factor vs. signal power using our nonlinearity decoupling method [8] in the DM link for the homogeneous setup. The performance is averaged over 10 seeds, each corresponding to random carrier states of polarization (SOP) of the transmitted channels. In [4] and [5] it has been shown that pathological cases of all-aligned PDL elements such as $\left(\vartheta_{i}, \varepsilon_{i}\right)=(0,0)$ and $\left(\vartheta_{i}, \varepsilon_{i}\right)=(\pi / 4,0)$ are the worst case of PDL in linear and nonlinear regimes, respectively. Hence, in order to understand the interaction of PDL with Kerr effect in a simple way, each nonlinear effect was analyzed separately in three scenarios: i) without PDL (solid lines), ii) with aligned PDL sections having $\rho_{i}=10 \log _{10} \frac{(1+\Gamma)}{(1-\Gamma)}=0.25 \mathrm{~dB}$ and $\left(\vartheta_{i}, \varepsilon_{i}\right)=(0,0)$ (dotted lines) or iii) $\left(\vartheta_{i}, \varepsilon_{i}\right)=(\pi / 4,0)$ (dashed lines). The curve labeled WDM corresponds to the real case including all nonlinearities.

We start analyzing the linear regime, i.e., the initial increasing part of the Q-factor curves. Here in all cases the worst PDL is with $\left(\vartheta_{i}, \varepsilon_{i}\right)=(0,0)$ where the polarization dependent SNR degradation induced by PDL is maximum and cannot be compensated by the DSP [4]. Moving to the nonlinear regime (decreasing part of the Q-factor curve), the behavior of SPM and XPM curves can be explained by looking at the total instantaneous power after the generic $i$ th PDL element:

$$
\begin{aligned}
P_{\text {out }}(t)= & \left|v_{X}^{(i)}\right|^{2}+\left|v_{Y}^{(i)}\right|^{2} \\
& +\Gamma \cos 2 \vartheta \cos 2 \varepsilon\left(\left|v_{X}^{(i)}\right|^{2}-\left|v_{Y}^{(i)}\right|^{2}\right) \\
& +\Gamma \cos 2 \vartheta \sin 2 \varepsilon\left(2 \mathrm{I}_{\mathrm{m}}\left\{v_{X}^{(i)}\left(v_{Y}^{(i)}\right)^{*}\right\}\right) \\
& +\Gamma \sin 2 \vartheta\left(2 \mathrm{R}_{\mathrm{e}}\left\{v_{X}^{(i)}\left(v_{Y}^{(i)}\right)^{*}\right\}\right) .
\end{aligned}
$$

For phase modulated signals $\left|v_{X}^{(i)}\right|^{2}$ and $\left|v_{Y}^{(i)}\right|^{2}$ remain almost constant along propagation in DM systems. Consequently, at $\varepsilon=0$ only the last term in (2) matters, especially when $\vartheta=\pi / 4$ [5]. Unfortunately, this term is data dependent and thus has wild variations in time [7] that enhance nonlinear distortions. Generalizing over the Poincare sphere, this effect is maximum when the 3D Stokes vector of the PDL eigenvector lays on the polarization plane of the input PDM-QPSK signal.

The $\vartheta$-dependence does not appear for XPM because the transmitted SOPs of the interfering channels are randomly oriented with respect to the central channel, thus removing 


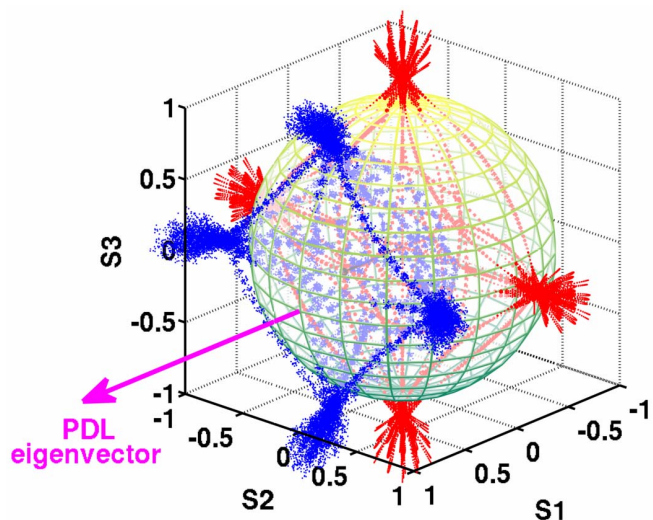

Fig. 3. Time-sampled Stokes vector of a PDM-QPSK signal before (red) and after (blue) propagation through a lumped PDL element with $\rho=5 \mathrm{~dB}$ and $(\vartheta, \varepsilon)=(0,0)$. Poincaré sphere shown for reference.

on average the dependence on the absolute reference system of the PDL axes (i.e., on $\vartheta$ ).

For XPolM the interplay with PDL is different. Because each PDL element is a partial polarizer, the pattern-induced temporal fluctuations of the SOP are reduced, hence reducing XPolM. This can be easily observed from Fig. 3, which depicts the time trajectory of the Stokes vector of a PDM-QPSK signal before and after propagation through a PDL element with $(\vartheta, \varepsilon)=(0,0)$ and $\rho=5 \mathrm{~dB}$. The re-polarization induced by the PDL eigenvector is clearly understood by the reduced distance in Stokes space of the four output PDM-QPSK symbol clouds. Fig. 2 (bottom, left) indicates that the linear penalty due to PDL is more than compensated by the reduction of XPolM at large powers. Overall, the beneficial impact of PDL on XPolM is not visible in the real WDM curve, an indication that SPM and XPM cannot be neglected when PDL is present. Moreover, the worst case Q-factor of the WDM case shows $1 \mathrm{~dB}$ of penalty with respect to the no PDL case at any power, although for different $\vartheta$, i.e., $\vartheta=\pi / 4$ in nonlinear regime and $\vartheta=0$ in linear regime.

Moving to the hybrid scenario, we replaced PDM-QPSK interfering channels with $10 \mathrm{~Gb} / \mathrm{s}$ OOK having average power locked $4 \mathrm{~dB}$ below that of the central $112 \mathrm{~Gb} / \mathrm{s}$ PDM-QPSK channel, in order to ensure equal performance for both modulation formats. Fig. 2 (center column) shows the average Q-factor when applying the nonlinear decoupling method. This setup has scalar XPM as the dominant nonlinear distortion because PDM-QPSK suffers the intensity fluctuations of OOK neighbors [10]. Since $v_{Y}^{(i)}=0$, only the first of PDL-related terms in (2) survives, causing a $\vartheta$-dependent coupling of XPM with PDL that can be detrimental when $\vartheta=0$ or beneficial when $\vartheta=\pi / 2$. However, the PDL/XPM interaction is in any case masked by the random orientation of the transmitted SOP of the interfering channels. XPolM is now a secondary impairment and does not show improvements with PDL, because the PDL polarizing effect has minor importance with OOK neighbors.

Fig. 2 (right column) shows the average Q-factor for the homogeneous setup transmitted on the DU link. In this scenario, dispersion induces strong signal fluctuations, hence

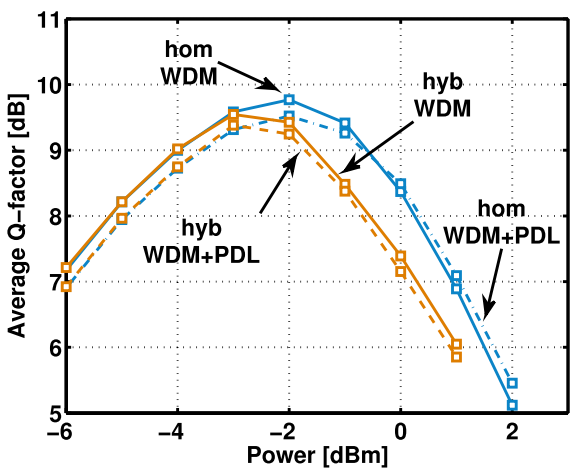

Fig. 4. Average performance in both hybrid and homogeneous scenarios for WDM propagation on the $20 \times 100 \mathrm{~km}$ DM link. Propagation without PDL or with $\rho_{\mathrm{rms}}=2.25 \mathrm{~dB}$ (PDL elements with random orientation).

reducing the importance of PDL-related terms in (2), as shown by the $\vartheta$-independent Q-performance of both SPM and XPM in nonlinear regime. XPolM coupling with PDL follows similar trends as ASE noise in linear regime, suggesting that XPolM behaves like an additive white noise. Overall, PDL worsens the Q-factor by about $1 \mathrm{~dB}$.

However in realistic setups the eigenvectors of PDL elements are randomly oriented, hence the pathological cases analyzed in Fig. 2 are very unlikely [4], [5]. In real setups the total cumulated PDL has a Maxwellian distribution with root mean square (rms) value related to $\rho_{i}$ by $\rho_{\text {rms }}=\rho_{i} \cdot \sqrt{N}$, with $N$ the number of spans. Fig. 4 reports the impact on the average Q-factor by randomly-oriented PDL elements for both homogeneous and hybrid scenarios on DM link. The performance is averaged over 10 seeds, each corresponding to random carrier SOP and PDL orientations $\left(\vartheta_{i}, \varepsilon_{i}\right)$. It can be noted that, in absence of nonlinearity, PDL yields a penalty of $0.3 \mathrm{~dB}$ on average Q-factor, as shown by the gap in linear regime between solid and dashed curves.

\section{Q-FACTOR Distribution}

A more interesting description of the impact of realistic PDL is given by the distribution of the Q-factor. To correctly estimate the performance distribution it is necessary to distinguish between input random variables (RVs) that do not vary during a BER measurement, i.e., non-ergodic RVs, and those that do, i.e., ergodic. The distribution of the Q-factor must account for the randomness of the non-ergodic RVs only, while the impact of the ergodic RVs must be averaged in the BER measurement. In our case, non-ergodic RVs were represented by carrier SOP and PDL orientations $\left(\vartheta_{i}, \varepsilon_{i}\right)$, while ergodic ones were ASE and symbol patterns. Thus the correct procedure for Q-factor probability mass function (PMF) [11] estimation requires two nested cycles: an inner one on ergodic RVs and an outer one on non-ergodic RVs. Besides correctly dealing with input RVs, it is necessary to avoid the Q-factor PMF being corrupted by the MC uncertainty in the inner cycle on ergodic RVs. Since MC error is independent of the non-ergodic RVs, it substantially adds its variance to the true variance of the Q-factor PMF. Therefore we must ensure that the MC variance in the ergodic inner cycle is much smaller than the variance of the overall PMF. We verified that for our system, counting at 

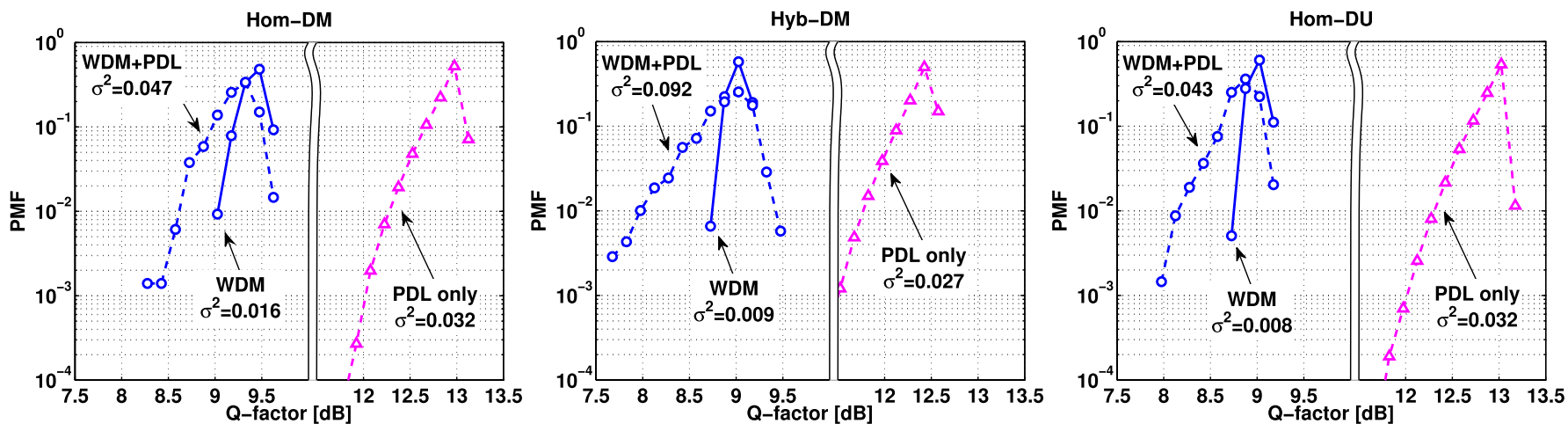

Fig. 5. Q-factor PMF for WDM propagation, without PDL (200 seeds) or with $\rho_{\mathrm{rms}}=2.25 \mathrm{~dB}$ (700 seeds, PDL elements with random orientation), and with only PDL $\left(10^{5}\right.$ seeds). Bin size $0.15 \mathrm{~dB}$. (Left) Homogeneous setup on the $20 \times 100 \mathrm{~km}$ DM link with Power $=-1 \mathrm{dBm}$. (Center) Hybrid setup on the $20 \times 100 \mathrm{~km}$ DM link with Power $=-1.5 \mathrm{dBm}$. (Right) Homogeneous setup on the $25 \times 100 \mathrm{~km}$ DU link with Power $=0 \mathrm{dBm}$. The outage probability at FEC Q-threshold of $8.5 \mathrm{~dB}$ is the sum of the PMF values below $\mathrm{Q}=8.5 \mathrm{~dB}$.

least 400 errors in absence of PDL and 300 errors with PDL was enough for an accurate overall PMF estimation.

Fig. 5 shows the estimated PMF of the Q-factor both in presence and absence of PDL for both homogeneous and hybrid WDM systems propagated in the DM link and for the homogeneous system propagated in the DU link. The PMF was estimated by iterating on different random seeds of the nonergodic RVs, and working $1 \mathrm{~dB}$ above the optimal power, i.e., with: $\mathrm{P}=-1 \mathrm{dBm}$ (optical SNR $(\mathrm{OSNR})=17 \mathrm{~dB} / 0.1 \mathrm{~nm}$ ) for the homogeneous DM setup; $\mathrm{P}=-1.5 \mathrm{dBm}(\mathrm{OSNR}=$ $16.5 \mathrm{~dB} / 0.1 \mathrm{~nm}$ ) for the hybrid $\mathrm{DM}$ setup; $\mathrm{P}=0 \mathrm{dBm}$ $(\mathrm{OSNR}=17 \mathrm{~dB} / 0.1 \mathrm{~nm})$ for the homogeneous DU setup. From Fig. 5 we can see that without PDL the Q-factor distribution is very narrow, indicating that the carrier SOP has little impact on it. Turning PDL on remarkably enlarges such a distribution, leading to an increased outage probability. For instance, assuming a forward error correction (FEC) Q-threshold of $8.5 \mathrm{~dB}$, in presence of PDL the probability of an outage event, i.e. Q-factor lower than $8.5 \mathrm{~dB}$, is $2.8 \cdot 10^{-3}$ for homogeneous and $1.2 \cdot 10^{-1}$ for hybrid systems on the DM link, $6.8 \cdot 10^{-2}$ for the homogeneous setup on DU link, while the outage probability without PDL is essentially zero.

Fig. 5 also shows, for each setup, the Q-factor PMF in absence of nonlinearity (PDL-only) with $\rho_{\mathrm{rms}}=2.25 \mathrm{~dB}$, obtained with the reverse channel method (RCM) [1] (and double-checked when feasible by MC simulations). We observe that the interaction between PDL and nonlinearity yields a Q-factor variance $\sigma^{2}$ at least 1.4 times larger with respect to the PDL-only case. We find that, contrary to [6], [7], the Q-factor PMF does not shrink with both PDL and nonlinearity, and always has a larger variance than the PDL-only case. The PDL-only PMF in [7, Fig. 2] is not backed by experiments, and the maximum Q-factor exceeds that predicted by RCM in absence of PDL. We suspect poor accuracy in the MC Q-factor estimation, which artificially broadens the PMF.

\section{CONCLUSION}

By decoupling the Kerr nonlinearities, namely SPM, XPM and XPolM, we investigated their interplay with PDL in dispersion-managed optical systems, showing different interactions between phase and polarization effects. We also showed that the presence of realistic random PDL has little impact on average Q-factor in both dispersion-managed and dispersion-unmanaged links, but makes a remarkable difference on Q-factor distribution. As a result, while we did not observe outage events without PDL, at an rms PDL of $2.25 \mathrm{~dB}$ we estimated an outage probability $6.8 \cdot 10^{-2}$ for the DU link at FEC Q-threshold of $8.5 \mathrm{~dB}, 2.8 \cdot 10^{-3}$ for the DM-homogeneous setup and $1.2 \cdot 10^{-1}$ for the DM-hybrid setup. Besides, the PDL-nonlinearity interplay gives a Q-factor distribution at least 1.4 times broader than the PDL-only case.

\section{REFERENCES}

[1] Z. Tao, L. Dou, T. Hoshida, and J. Rasmussen, "A fast method to simulate the PDL impact on dual-polarization coherent systems," IEEE Photon. Technol. Lett., vol. 21, no. 24, pp. 1882-1884, Dec. 15, 2009.

[2] M. Shtaif, "Performance degradation in coherent polarization multiplexed systems as a result of polarization dependent loss," Opt. Express, vol. 16, no. 18, pp. 13918-13932, Sep. 2008.

[3] C. Xie, "Polarization-dependent loss induced penalties in PDM-QPSK coherent optical communication systems," in Proc. OFC/NFOEC San Diego, CA, USA, Mar. 2010, pp. 1-3, paper OWE6.

[4] O. Vassilieva, I. Kim, and T. Naito, "Systematic investigation of interplay between nonlinear and polarization dependent loss effects in coherent polarization multiplexed systems," in Proc. 36th ECOC, Torino, Italy, Sep. 2010, pp. 1-3, paper P4.08.

[5] Z. Tao, L. Li, T. Hoshida, and J. Rasmussen, "Interaction between PDL and intra-channel nonlinearity in dual polarization systems," in Proc. 16th OECC, Kaohsiung, Taiwan, Jul. 2011, pp. 224-225, paper 6B3_2.

[6] O. Vassilieva, I. Kim, and M. Sekiya, "Statistical analysis of the interplay between nonlinear and PDL effects in coherent polarization multiplexed systems," in Proc. ECOC, Amsterdam, The Netherlands, Sep. 2012, pp. 1-3, paper We.3.C.4.

[7] O. Vassilieva, S. Oda, T. Hoshida, J. Rasmussen, and M. Sekiya, "Experimental investigation of the statistics of the interplay between nonlinear and PDL effects in coherent polarization multiplexed systems," in Proc. OFC, Anaheim, CA, USA, Mar. 2013, pp. 1-3, paper OM3B.6.

[8] A. Bononi, P. Serena, N. Rossi, and D. Sperti, "Which is the dominant nonlinearity in long-haul PDM-QPSK coherent transmissions?" in Proc. 36th ECOC, Torino, Italy, Sep. 2010, pp. 1-3, paper Th.10.E.1.

[9] P. Serena, M. Bertolini, and A. Vannucci. (2009). Optilux Toolbox [Online]. Available: http://www.optilux.sourceforge.net

[10] A. Bononi, N. Rossi, and P. Serena, "Transmission limitations due to fiber nonlinearity," in Proc. OFC/NFOEC, Los Angeles, CA, USA, Mar. 2011, pp. 1-3, paper OWO7.

[11] A. Papoulis, Probability, Random Variables, and Stochastic Processes, 3rd ed. New York, NY, USA: McGraw-Hill, 1991 